\title{
LA REGULACIÓN AFECTIVA EN LA CONFORMACIÓN DE LA PERSONALIDAD. Estudio en escolares de 5 a 8 años*
}

\author{
AFFECT AND ITS REGULATION IN PERSONALITY ORGANIZATION. \\ Study on school children, five to eight years old
}

\author{
Delfina Miller \\ Universidad Católica del Uruguay
}

\begin{abstract}
Resumen: Este trabajo profundiza, desde la perspectiva psicoanalítica, en la regulación afectiva y su incidencia en la organización de la personalidad, a partir de una investigación tanto conceptual como empírica. Se utilizó un diseño cuanti-cualitativo, sobre una muestra de 82 niños montevideanos, provenientes de tres medios socioeconómicos diferentes (alto, medio, bajo), La media de edad fue 6,2 años, con una prevalencia del $64 \%$ de varones. En la muestra se evidenció una alta prevalencia (18,9\%) de niños con disregulación, provenientes en su mayoría del medio socioeconómico más carenciado (78,5\%), de familias monoparentales y con antecedentes psiquiátricos. Según este estudio, la disregulación se asocia a importantes alteraciones en el desarrollo especialmente sueño, aprendizaje, adquisición de hábitos y empatía, generando dificultades en todas las áreas de desarrollo. Los niños con disregulación afectiva evidencian muy escasos afectos positivos y prácticamente no muestran miedo ni tristeza, tanto en sus juegos como en sus reacciones psicofisiológicas.
\end{abstract}

Palabras Clave: regulación afectiva, infancia, personalidad

\begin{abstract}
The present study seeks to deepen in affect regulation and its impact on the organization of personality, from a psychoanalytical perspective, based on both conceptual and empirical research. A qualitative-quantitative design was employed, on a sample of 82 montevidean children, belonging to three different socio-economic groups (low, medium and high) with a mean age of 6.2 years; $64 \%$ of them were males. The study showed a high prevalence of emotionally dysregulated children (18.9\%), from the most disadvantaged socioeconomic environment (78.5\%) part of single parent families, with background of psychiatric disorders.According to this thesis, emotional dysregulation is associated with important developmental disturbances related specially to sleep, learning processes and the acquisition of skills and empathy.Children with emotional dysregulation show very low positive affect and practically do not display any signs of fear or sadness both while playing and in their psychophysiological reactions.
\end{abstract}

Keywords: affective regulation, childhood, personality

\begin{abstract}
* Este trabajo recoge parte de los resultados del estudio realizado para obtener el grado de doctor en la Facultad de Psicología de la Universidad de Buenos Aires, bajo la tutoría del Prof. Dr. Ricardo Bernardi, sobre "La incidencia de la regulación afectiva en la personalidad, desde una perspectiva psicoanalítica".
\end{abstract}

\section{Introducción}

A través de este trabajo procuraré profundizar, desde una perspectiva psicoanalítica, en la regulación afectiva y su incidencia en la organización de la personalidad, ofreciendo los aportes de una investigación tanto conceptual como empírica que fuera parte de mi tesis de Doctorado.

El interés del tema se sustenta por un lado en la permanente y renovada atención de la Psicología y del Psicoanálisis en los afectos y en la incidencia de su disregulación ${ }^{1}$ en la conformación de la patología, y por otro lado en el incremento de las consultas, en especial en la clínica infantil, por una sintomatología caracterizada por lo que podría considerarse una afectividad disregulada, que se expresa

\footnotetext{
1. Debemos de tener en cuenta que el término "disregulación" o "desregulación" no existen como tales de acuerdo al Diccionario de la Real Academia Española. Sin embargo se trata de términos que se han vuelto de uso frecuente en función de los múltiples estudios que se han realizado al respecto, como fácilmente lo comprobamos en la bibliografía citada. Provienen de la traducción del término inglés "dysregulation". A los efectos de esta tesis usaré entonces la expresión "disregulación" en atención a efectuar la traducción del inglés más específica.
} 
clínicamente en forma de agresividad, ansiedad o depresión.

Los estudios acerca de la regulación de los afectos me han llevado a aunar en la consideración de este fenómeno diversas perspectivas: la biológica y la psíquica, la intrapsíquica y la intersubjetiva, la individual y la social, integrando así diferentes variables que tienen incidencia tanto en el desarrollo como en la conformación de la psicopatología.

En este sentido, y con la intención de comenzar a sistematizar los datos provenientes de la clínica promoví un relevamiento de los casos atendidos en el período 2003-2008, en una policlínica de atención psicológica de la Facultad de Psicología de la Universidad Católica del Uruguay. En él pude constatar varios aspectos que resultaron de interés, teniendo en cuenta que el mayor número de consultas eran de niños en edad escolar, con graves problemas psicosociales, siendo la mayoría de las derivaciones provenientes del entorno escolar.

El motivo de consulta más frecuente era la agresividad (afectividad disregulada) seguida de los problemas en el rendimiento escolar (evidencia de escasa presencia y uso de herramientas cognitivas). Pude comprobar que frecuentemente la agresividad correspondía a una manifestación conductual propia de un bajo desempeño intelectual en el que predominaba la impulsividad, la disregulación afectiva, el escaso uso de herramientas cognitivas y la ausencia de empatía.

Es a partir de este primer relevamiento que surge la necesidad de profundizar la investigación acerca del tema, dando prioridad a la disregulación del afecto de la que los síntomas daban cuenta, haciendo especial énfasis en la dinámica interna que sostenía y organizaba la afectividad.

\section{Relevancia del tema estudiado}

Si bien la dificultad en la regulación afectiva, concretamente de la agresividad en sus diversas manifestaciones, es hoy uno de los motivos frecuentes de consulta en la infancia, son claramente muy escasos los trabajos teóricos psicoanalíticos referidos a estas manifestaciones en la edad escolar, y más escasas aún las investigaciones empíricas al respecto, en especial en nuestro medio.
La mayor parte de las investigaciones encontradas se han centrado en los primeros años de vida y no en la edad escolar. Muchos investigadores (por ejemplo Bretherton, 1984; Emde, Wolf \& Oppenheim, 2003; Fonagy, 2004; Piaget, 1961; Slade \& Wolf, 1994) se focalizan en el estudio de niños pequeños, planteando que la consideración de estos aspectos en los primeros momentos de vida resulta de fundamental importancia. En los escolares los estudios parecen centrarse más en las habilidades y estrategias de aprendizaje (Fonagy \& Target, 2002; Emde et al., 2003) y no en la afectividad y su regulación.

Resulta, entonces, de gran utilidad comprender qué mecanismos psíquicos sostienen las condiciones de la afectividad disregulada, en una edad en la que se consolida el concepto de sí mismo, se establecen pautas de relacionamiento con pares que se mantienen y que contribuyen a la imagen de sí, en la que el funcionamiento en el grupo de pares marca pautas de identificación y competencia que serán claves en el establecimiento de la autonomía y en la qué el desarrollo de herramientas cognitivas se vuelve de capital trascendencia (Sroufe, 2000).

\section{Marco Teórico}

El concepto de afecto resulta fundamental para el psicoanálisis ya desde los primeros trabajos de Freud. Siguiendo sus ideas, observamos dos tendencias:

- Tomando en consideración sus planteos en "Lo Inconciente" (Freud, 1915/1980), un afecto es la percepción consciente de un proceso inconsciente de descarga pulsional. Los afectos están considerados entonces como energía en busca de descarga y vinculados específicamente al ello.

- Por otro lado en "Inhibición, Síntoma y Angustia" (Freud, 1926/1980) considera a los afectos como señales que pasan a través del control del yo. Señales de angustia que previenen al individuo de verse sorprendido por su propia indefensión.

Podemos entonces preguntarnos ¿surge el afecto de un flujo desorganizador inconsciente o es un mensaje indicador de las características del estímulo?

La segunda tendencia, que considera los afectos como señales, ha sido desarrollada especialmente por la psicología de las re- 
laciones objetales y la psicología del self, así como por los estudios de la regulación afectiva, especialmente por los teóricos del apego. Estudios contemporáneos destacan la función de los afectos como indicadores de la importancia que el sujeto da a los diferentes estímulos, condicionando tanto su percepción como su respuesta conductual. Son considerados experiencias subjetivas que a la vez que nos hacen "sentir" condicionan también nuestro actuar (Bernardi, 2007; Emde, 1988). Para estos autores estas experiencias subjetivas se consolidan en estructuras internas que van conformando el sustrato de la personalidad.

Otros psicoanalistas sostienen que sólo la primera puede considerarse auténticamente psicoanalítica (Green, 1999) ya que la segunda al poner el énfasis en el control del yo descuidaría el lugar del inconsciente en la generación de afectos. Este planteo, nos lleva a considerar qué incidencia y que características se le atribuye a lo inconsciente así como a los procesos cognitivos, en relación a la afectividad, vieja controversia entre afecto y representación, entre idea y emoción, que puede hoy traducirse a la pregunta acerca de ¿cuánto incide el afecto en la cognición y cuánto ésta en el afecto?

Estudios contemporáneos nos muestran que ambos procesos son difícilmente separables ya que "la cognición está al servicio del afecto y ésta inspira la cognición" (Sroufe, 2000, p.160).

Asimismo, las investigaciones actuales que reúnen los conocimientos psicoanalíticos con los provenientes de la biología o de los estudios acerca del desarrollo, muestran que el afecto es considerado como una fuente independiente de conocimiento tanto de uno mismo como del entorno (Damasio, 2010), suponiendo un modo independiente de evaluación (Ledoux, 1999), en el que una gran parte de su procesamiento y regulación se daría automáticamente, fuera de la conciencia y en ocasiones con independencia de su representación. Emde (1998), por su parte, también plantea que habría una evaluación afectiva inmediata, proceso que ocurriría de forma espontánea, automática, y la mayoría de las veces, subliminal.

Esta idea del afecto proveyéndonos de información está también presente en Bion y Winnicott (como se citó en Green, 1999), así como en Fonagy (Fonagy, 2004) quienes consideran el afecto como la base del desarrollo primario, enfatizando en la importancia de su regulación, aún cuando se puede ver una gran disparidad entre el rol marginal que se le ha concedido dentro de la teoría psicoanalítica y su enorme importancia en la clínica, en su vinculación con los procesos inconscientes, los deseos, imbricados dentro del proceso de desarrollo (Fonagy \& Target, 2002).

El estudio más sistemático así como la investigación en la regulación de los afectos se originaron en la Psicología del Desarrollo, (Gross, 2007) y ahora se están extendiendo tanto a la Psicología del adulto (Gross, 2007) como a la Psicopatología (Bradley, 2000) cubriendo diversas perspectivas.

Podemos definir la Regulación Afectiva como la capacidad del yo para modular los estados afectivos. Dicha capacidad se sustenta en una significación experiencial que va más allá de la comprensión intelectual ya que a la vez que habilita la mentalización se ve luego condicionada por ella. En este sentido la "afectividad mentalizada" estaría en la base de la capacidad madura para regular los afectos y para descubrir el significado subjetivo de los estados afectivos propios. Su importancia radica en que juega un papel fundamental en el desarrollo del reconocimiento y manejo de uno mismo, así como en la adaptación del sujeto a su entorno (Fonagy, Gergely, Jurist, \& Target 2004).

La experiencia del afecto constituye la base a partir de la cual la mentalización puede irse desarrollando, pero sólo en el contexto de una relación continuada y segura. En estos procesos que tienden a la transformación de los montajes sensorio-motores en representantes pulsionales es fundamental el papel del otro originario, así como el de los muchos otros que incidirán en la estructuración psíquica. Puede decirse que el adulto opera, desde los primeros vínculos con el niño, como un conmutador que transforma los esquemas sensorio-motores, con los cuales nace el bebé, en pulsiones que se ponen de manifiesto como afectos y marcas significantes.

Resulta de especial interés, en este punto, tomar en consideración la teoría del apego desarrollada por John Bowlby (1986), así como la propuesta de Sroufe (2000) quien reconceptualizó la teoría del apego en términos de regulación del afecto, planteando que las personas con apego seguro, las cuales han internalizado las capacidades de autorregula- 
ción, contrastan con aquellas que en etapas precoces, o no han llegado al nivel adecuado de regulación del afecto (evitativas) o han sobrepasado este nivel (resistentes). En este contexto el apego sería conceptualizado como un proceso intersubjetivo que condicionaría el paso a una vida mental compleja, así como a un sistema conductual específico.

Los afectos serán los motivadores de las interacciones tempranas, interacciones que se irán internalizando y en su internalización promoverán las representaciones tanto del self como del objeto. Otto Kernberg (1994) acuerda con esta posición y plantea que una vez que la organización psíquica se ha consolidado, sus activaciones se deberán a estados afectivos. Estos estados afectivos incluyen relaciones objetales internalizadas y una básica representación de sí mismo que se vincula a una particular representación del objeto.

A partir de lo expuesto, podemos concluir que los afectos aparecen como procesos evaluativos, que arraigan en la biología, son activos y adaptativos, incluyen cogniciones, y operan tanto consciente como inconscientemente, a través de códigos simbólicos y subsimbólicos, previos al desarrollo del pensamiento (Bucci, 2003), reuniendo lo intrapsíquico con lo intersubjetivo con un objetivo integrador, al organizar el funcionamiento mental y la conducta (Emde, 1988).

Desde diferentes perspectivas se sostiene que el individuo adquiere a lo largo de su vida diversas estrategias (defensas, atribuciones, estrategias cognitivas) que le permiten regular sus afectos así como sus vínculos, dependiendo de las características personales, la interrelación con los cuidadores y las experiencias vivenciadas. Estos mecanismos se utilizarán con mayor o menor frecuencia, y de forma más o menos flexible, conformando la estructura de la personalidad, como lo ha descrito Main (como se citó en Bradley, 2000).

Este proceso se enmarca tanto en la vertiente intersubjetiva como en la capacidad del niño para captar, registrar y expresar. Es decir que un aspecto esencial será la permeabilidad del niño para detectar, comunicar y responder a los diferentes estímulos, así como el poder hacerlo a través de diferentes estados afectivos. Esta disponibilidad será tanto o más importante que la que tenga el cuidador. Ambas son las que darán lugar al encuentro o al desencuentro. La rigidez o la imposibilidad, de una de las partes, tanto en el registro como en el procesamiento o en la comunicación, será entonces un fuerte predictor de patología. El planteo freudiano de las series complementarias nos resulta de especial utilidad en este aspecto.

Entonces, podemos decir que los afectos acompañan y aparecen ya desde los primeros momentos de la existencia humana, y será su identificación, diferenciación y en definitiva su regulación lo que les dará su lugar en el desarrollo de la personalidad. Diversos afectos coexisten y cada uno de ellos sostiene una disposición particular para el reconocimiento y la adaptación del niño a su entorno. Teorías neuropsicológicas actuales presuponen la existencia de un almacenamiento de recuerdos afectivos en el cerebro límbico, que permite la reactivación de aspectos no solamente cognitivos sino también afectivos de la experiencia vivida, en especial aquellos aspectos subjetivos que colorearon afectivamente dicha experiencia (Arnold 1970). Al decir de Cole, Luby y Sullivan (2008), al ser capitales para el desarrollo, también vuelven al niño más vulnerable, aún cuando sean básicamente adaptativos.

Debemos igualmente considerar que tanto los planteos teóricos como las investigaciones empíricas nos muestran la relación entre la dificultad de regulación, el aumento de afectos negativos y el pasaje a la acción sin el suficiente respaldo representacional. Son éstas las condiciones que estarían en la base de las manifestaciones agresivas, de acuerdo a lo mencionado en la introducción, en dónde se caracterizaba la agresividad como una evidencia de afectividad disregulada, manifestación conductual propia de un bajo desempeño intelectual en el que predomina la impulsividad, las bajas herramientas cognitivas y la ausencia de empatía.

Podemos entonces decir que cuando no se logra una adecuada regulación nos encontramos frente a una afectividad que invade, que no logra ser una "señal" al no ser adecuadamente significada ni procesada, que por su misma fuerza puede llevar a vivirlo todo como una amenaza o un ataque. El sujeto deberá entonces mantenerse en guardia consumiendo así buena parte de su energía. No quedaría entonces energía para otras funciones, que se verían claramente afectadas. 


\subsection{La agresividad como expresión de afecto disregulado}

Focalicemos ahora en la consideración de la agresividad considerándola como una expresión paradigmática de afecto. Siendo la base de la fortaleza, de la iniciativa, de la ambición, de la decisión y el valor, la agresividad es una disposición innata del comportamiento que va a ser disparada por estímulos externos o internos que estarían en relación tanto con el crecimiento como con la frustración y al servicio de la lucha del sujeto, y podría decir del yo, por su conservación y fortalecimiento. En este sentido la agresividad como respuesta natural tendría su origen en la defensa de uno mismo, de su territorio, de su posicionamiento o jerarquía dentro del mismo.

En su expresión (autoagresividad o heteroagresividad) aparecería como no patológica en la medida en que resulta adaptada, acorde al estímulo que la dispara y surge en respuesta a un estímulo específico, no manteniéndose una vez que se ha respondido a éste. En la medida en que se transforma en una manifestación caracterológica crónica se volvería patológica (Kernberg, 1994).

Podemos, a su vez, considerarla como una actividad-descarga, como una pulsión o como un afecto. En tanto actividad-descarga, vuelvo nuevamente a lo planteado acerca de los afectos: son varios los autores que nos hablan de un ejercicio cuya finalidad sería simplemente liberar tensión (Freud, 1926/1980; Winnicott, 1990; Kernberg, 1994). Aquí estamos haciendo referencia a respuestas muy primitivas, carentes de un sustrato representacional, que se darían de forma espontánea y con escasa conciencia.

En la medida en que el sujeto significa, registra y relaciona, la agresión va de alguna manera consolidándose como pulsión y en su internalización más permanente, en su integración a la trama de fantasías característica del sujeto, podrá ser considerada como un afecto.

Deben considerarse igualmente dos aspectos de la agresividad: el acto agresivo por un lado y la fantasia que lo acompaña por otro. Me interesa especialmente esta última, sostenida en representaciones objetales internalizadas, a la que se puede diferenciar de lo que sería simple descarga o impulsividad. A su vez, y en relación a la consideración de la fantasía que acompaña las manifestaciones visibles, qui- siera especificar nuevamente que agresividad (fuerza, impulso) y agresión (manifestación hostil) no son inherentemente patológicas sino que pueden ser parte de una respuesta saludable. Lo importante es diferenciar, más allá del acto agresivo, cuando la manifestación tiene como finalidad la destrucción o cuando está al servicio de alcanzar una meta (fortalecimiento o defensa). Es decir cuando está relacionada con un sentimiento de desprotección, desvalimiento o frustración y cuando busca simplemente la destrucción del objeto disfrutando o no con ello.

Desde los orígenes, el psicoanálisis considera estas diferentes opciones: la agresividad como fuerza vital al servicio de la conservación, el desarrollo o la defensa, o la agresividad como fuerza destructiva que puede ir o no asociada al placer. Al igual que he planteado anteriormente en referencia al afecto en general, no parece que la opción sea elegir entre estos orígenes o fines de la agresividad, sino tomarlos en cuenta para determinar en cada caso a que correspondería dicha manifestación. A esto puede agregarse que el sentido que tenga la agresividad va a resultar determinante en la conformación de la personalidad.

\subsection{Internalización y Externalización}

Vayamos ahora a las diferentes formas en las que se puede manifestar y regular el afecto y en especial la agresividad, para lo cual podemos retomar el planteo de la Introducción de este trabajo con respecto a la alta frecuencia de niños con disregulación afectiva caracterizada por una conducta agresiva en nivel clínico, expresada por síndromes tanto externalizantes (comportamientos manifiestos desajustados, agitación psicomotora, desobediencia y comportamiento oposicionista o delincuente) como internalizantes (enmascarando vivencias de desvalimiento, depresión, ansiedad).

Para ello y en función del destacado lugar que han tomado los términos Internalizante y Externalizante dentro de la psicopatología actual que considera la regulación de los afectos como primordial, quisiera comenzar por caracterizarlos tomando como referencia lo planteado por Western, Shedler, Bradley y DeFife (2011).

Con respecto a los sujetos que evidencian "síndromes externalizantes" suelen ser hostiles, expresando su agresividad directamente, 
en ataques de rabia o mostrándose críticos, controladores u oposicionistas. Sospechan de los otros, manifiestan conflictos con la autoridad y parecen siempre dispuestos a querellar. No muestran empatía, estando siempre pendientes de demostrar su valía. No reconocen sus carencias y culpan de ellas a otros. Tienen muy poco insight acerca de las motivaciones de su conducta y tienden a sentirse maltratados por los demás. No logran establecer vínculos profundos o estables. Su personalidad se enmarca dentro del tipo antisocial, psicopático, paranoide o narcisista.

Los sujetos con "síndromes internalizantes" son aquellos en los que predominan la depresión y la ansiedad, que suelen ser emocionalmente inhibidos y temerosos, socialmente vergonzosos y evitativos, a la vez que tienden a sentirse inadecuados y a culparse de sus dificultades. No se atreven a expresar sus afectos, deseos o impulsos. Suelen ser pasivos y sentirse desvalidos, impotentes, a merced de fuerzas ajenas a ellos. Permanecen rumiando sus problemas y a la vez que sienten un gran vacío, se les hace difícil expresar abiertamente su rabia hacia los otros y en cambio la expresan hacia ellos mismos. Su personalidad se enmarca dentro del tipo depresivo, ansioso, dependiente, evitativo y esquizoide.

Ahora bien, si pensamos en el mecanismo psíquico en juego, podríamos decir que la modalidad externalizante, con su recurso inmediato a la acción y a la descarga seguramente en pos de la regulación, parecería corresponder a un nivel más primario, a una trama representacional más pobre, frente a la imposibilidad de procesamiento de los estímulos que generan alguna reacción afectiva, como ya se planteó anteriormente.

Por su parte cuando el afecto, la agresividad, aparece unida a un síndrome internalizante, camuflando el desvalimiento y la minusvalía, regulando la reacción afectiva frente a ellos, parecería necesitar de un procesamiento mental mayor, unido a una cierta elaboración de la imagen de sí apoyada en una mayor introyección (aún cuando la imagen de sí sea deficitaria). Las relaciones de objeto irían más allá de la necesidad de satisfacción, las representaciones de objeto y de self estarían diferenciadas, ya que el sentimiento de desvalorización parecería requerir de una conciencia de self y de una cierta capacidad autorreflexiva, así como de una cierta evaluación de secuencias causales.
Aun cuando la internalización y la externalización implican mecanismos psíquicos diferentes, varias investigaciones (Achenbach, 1993; Cole et al., 2008) muestran que se presentan con una muy alta comorbilidad y que esta es sostenida justamente por la conducta agresiva.

Al presentarse juntas, nos estarán indicando que el sujeto no puede hacerse cargo de ciertos estímulos y la señal afectiva que marca su presencia, dispara o bien la inhibición (internalización) o bien la acción (externalización), en lugar de dar lugar a un procesamiento mental y a una elaboración de la sensación (señal afectiva) que le resulta intolerable, para lograr con ello lograr una respuesta más equilibrada.

Es en esta línea que se desarrollaron las investigaciones de Althoff, Retew, Ayer y HudZiak (2010); acerca de la Escala de Disregulación, que incluye conjuntamente la conducta agresiva y los síntomas depresivo-ansiosos, como manifestaciones de afectividad disregulada, y que demuestran las consecuencias sumamente negativas que esta asociación puede tener para el individuo (consumo de alcohol o drogas, suicidio, alteraciones graves en la personalidad en la adolescencia o adultez).

Por otro lado parece importante destacar la relación que permanentemente se ha comprobado tanto en la clínica como en los estudios teóricos, entre la agresividad y la necesidad de fortalecimiento e individuación, más allá de su connotación destructiva que parecería relacionada al fracaso en procesar el desvalimiento.

De acuerdo a lo que he planteado, resulta entonces deseable que el afecto se constituya en una señal a partir de la cual se pueda organizar la respuesta (Fonagy, 2004; Winnicott, 1990), esté en relación al estímulo que lo provoca, esté al servicio de la protección y el fortalecimiento del sujeto, le ayude en el reconocimiento de sí mismo, de sus herramientas y sus carencias, y que su intensidad no suma al sujeto en una situación de desvalimiento (Winnicott, 1990). Esto es lo que hace al afecto (más allá de su valencia) adaptativo (Cole et al., 2008; Fonagy, 2004).

Estas condiciones no se cumplen cuando el sujeto se siente desvalido, lo que lo lleva a interpretar los estímulos como amenazantes y en consecuencia a defenderse frente a ellos (agrediendo) o dejándose ganar por la indefensión (depresión). 


\section{Investigación}

Volvamos ahora, enriquecidos con estos planteos teóricos a la evidencia de la disregulación en los niños entendiendo por disregulación afectiva a la respuesta afectiva desadaptada basada en la ineficacia en la identificación, significación y procesamiento de las experiencias, que genera fallas en el reconocimiento de uno mismo y de los demás. Se evidencia a través de un aumento de la agresividad, la ansiedad, la depresión y los problemas atencionales.

\subsection{Objetivos}

Este trabajo tuvo como objetivo principal el estudio de la disregulación afectiva en niños de edades comprendidas entre 5 y los 8 años, provenientes de 3 centros de enseñanza de Montevideo, con distinto nivel socio económico (bajo, medio y alto).

Como objetivos específicos, para profundizar en la comprensión de la disregulación afectiva, caracterizada por la ineficacia en la identificación, significación y procesamiento de los estímulos que impiden una respuesta adaptada, me propuse:

- Evidenciar la prevalencia y caracterización de los niños disregulados.

- Comparar los niños disregulados con los niños no disregulados en cuanto a factores sociodemográficos y clínicos, analizando la posible asociación entre las variables clínicas y la historia de desarrollo.

- Analizar aquellos factores asociados con la disregulación, de modo de poder ofrecer un perfil de funcionamiento característico del grupo disregulado.

\subsection{Metodología}

Me resultó importante poder acceder a una metodología de investigación teórico-clínica aplicada, transversal, observacional, con un diseño cuanti-cualitativo, ya que reflejaba mi objetivo de realizar una evaluación en un momento determinado (transversal), haciendo especial referencia a los resultados (investigación clínica aplicada), a través de un diseño combinado (cuali-cuantiativo).

\subsection{Participantes}

82 escolares de Montevideo, de edades comprendidas entre los cinco y los ocho años, que concurren a tres escuelas representativas de diferentes medios socioeconómicos (bajo, medio y alto).

Criterios de exclusión: alteraciones graves en el funcionamiento psíquico del niño (Psicosis, Autismo, Retardo Mental).

\subsection{Instrumentos}

Los instrumentos que se aplicaron a dicha población con el objetivo de evaluar diferentes aspectos (comportamiento, vivencia subjetiva y correlatos psicofisiológicos), considerando el aporte de diferentes informantes (padres, maestros y niños) y sobre la base de la historia del desarrollo, fueron los siguientes:

- Historia de Desarrollo Protocolizada: Clásica historia del desarrollo que cubre desde el embarazo hasta el momento actual desde una perspectiva biopsicosocial.

- Child Behavior Check List (Achenbach, 1991): cuestionario realizado a los padres con el objetivo de conocer su impresión acerca de la manifestaciones conductuales y emocionales del niño.

- Teacher's Report Form (Achenbach, 1991): cuestionario a maestros acerca de las manifestaciones conductuales y emocionales del niño en relación a su desempeño en la escuela.

- Macarthur Story Stem Battery (Emde et al., 2003): técnica proyectiva que a través del planteo de situaciones dilemáticas, y en la evaluación tanto del juego como de la narrativa, busca profundizar en la dinámica psíquica del niño.

- FIRE (Miller et al., 2009): procedimiento de evaluación de juego naturalístico a partir de elementos estructurados y seleccionados por el investigador con el objetivo de inferir mecanismos de regulación del afecto.

- Prueba de Conductancia Dérmica: medición de la reacción (conductancia eléctrica de la piel) a estímulos (fotos) que evocan miedo, tristeza o agresividad.

Busqué a través de la aplicación de estos instrumentos confrontar los conceptos psicoanalíticos clásicos referidos al afecto y a su regulación con estudios que se sitúan en 
la zona de convergencia entre lo cognitivo, lo afectivo y lo comportamental, lo que me permitió evaluar dichos procesos en su intersección en la conformación de las estrategias de regulación afectiva.

Me enfoqué en la identificación, expresión y modulación de los afectos, así como en las condiciones de las representaciones de sí y de los objetos, su organización, la fantasía escenificada a través de la significación atribuida a los objetos (juguetes), a la situación misma de juego o a las situaciones planteadas, todo ello en relación con las performances en la realidad material evidenciadas tanto a través de las informaciones de padres y maestros como del vínculo transferencial, lo cual me permitió llegar a apreciaciones más profundas acerca de los mecanismos involucrados en la expresión disregulada de los afectos y, como veremos, especialmente de la agresividad.

\section{Resultados}

Vayamos ahora a la consideración de los resultados obtenidos:

Con respecto a la edad, la media de la muestra resultó de 6.2 años $(D E=.79)$ en un rango de 5 a 8, siendo la edad de los niños de la escuela 3 , de medio socioeconómico alto, significativamente menor que los de la escuela 2 , e medio socioeconómico medio $(p<.05)$.

Con respecto al sexo no se encontraron diferencias significativas en cuanto a su distribución por escuelas, ni tampoco en referencia a la composición familiar, más allá de que la Escuela 1 (población de más bajos recursos socio-económico-culturales) evidencia que solamente el $56 \%$ de los niños vive con ambos padres, frente a la escuela 2 (73\% ) y la $3(87 \%)$.

Resulta significativa la diferencia entre las escuelas en lo que respecta a los antecedentes de enfermedad mental, evidenciando la Escuela 1 una proporción mayor de antecedentes de enfermedad mental (33\%), $p<.05$, frente a un 3\% y un $5 \%$ de las escuelas 2 y 3 respectivamente. La Escuela 3 reporta el consumo de alcohol más alto (24\%).

Resultan también significativas las diferencias en lo que respecta a problemas de enseñanza y afectivos, siendo los problemas afectivos así como los de enseñanza significativamente mayores en la escuela $1,(p<.005)$.

Con respecto a los problemas emocionales y conductuales referidos por los padres (Child Behavior Check List) se evidencia una clara presencia de agresividad, seguida de depresión-ansiedad y problemas atencionales (Figura 1), siendo los varones los que eviden-

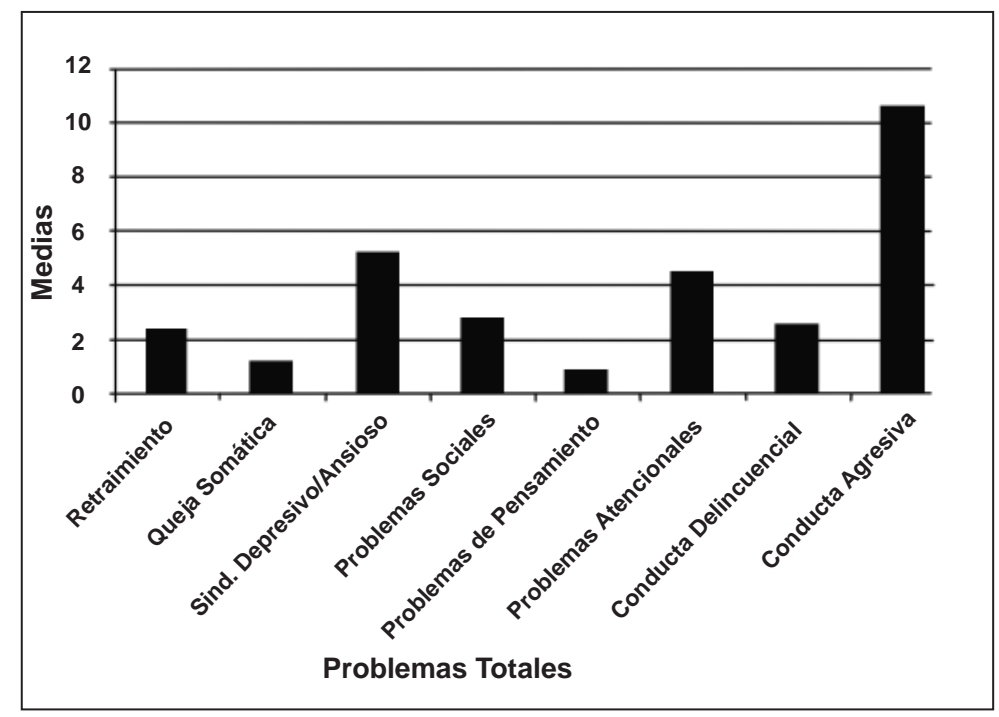

Figura 1. Problemas emocionales y conductuales referidos por los padres 
cian más dificultades, presentándose el mayor índice de problemas en la escuela de nivel socioeconómico bajo (Figura 2).

Con respecto a la prevalencia de niños disregulados nos encontramos con un $19 \%$ y una nueva prevalencia de los varones frente a las niñas y de la escuela 1 (de nivel socioeconómico bajo) frente a las otras escuelas.
Vayamos ahora a la consideración de las diferencias entre los niños disregulados y los no disregulados tal como lo refleja la figura 3. Allí nos encontramos con que los niños disregulados evidencian más problemas emocionales y de comportamiento en todas las áreas evaluadas y especialmente en agresividad, depresión-ansiedad y problemas atencionales.

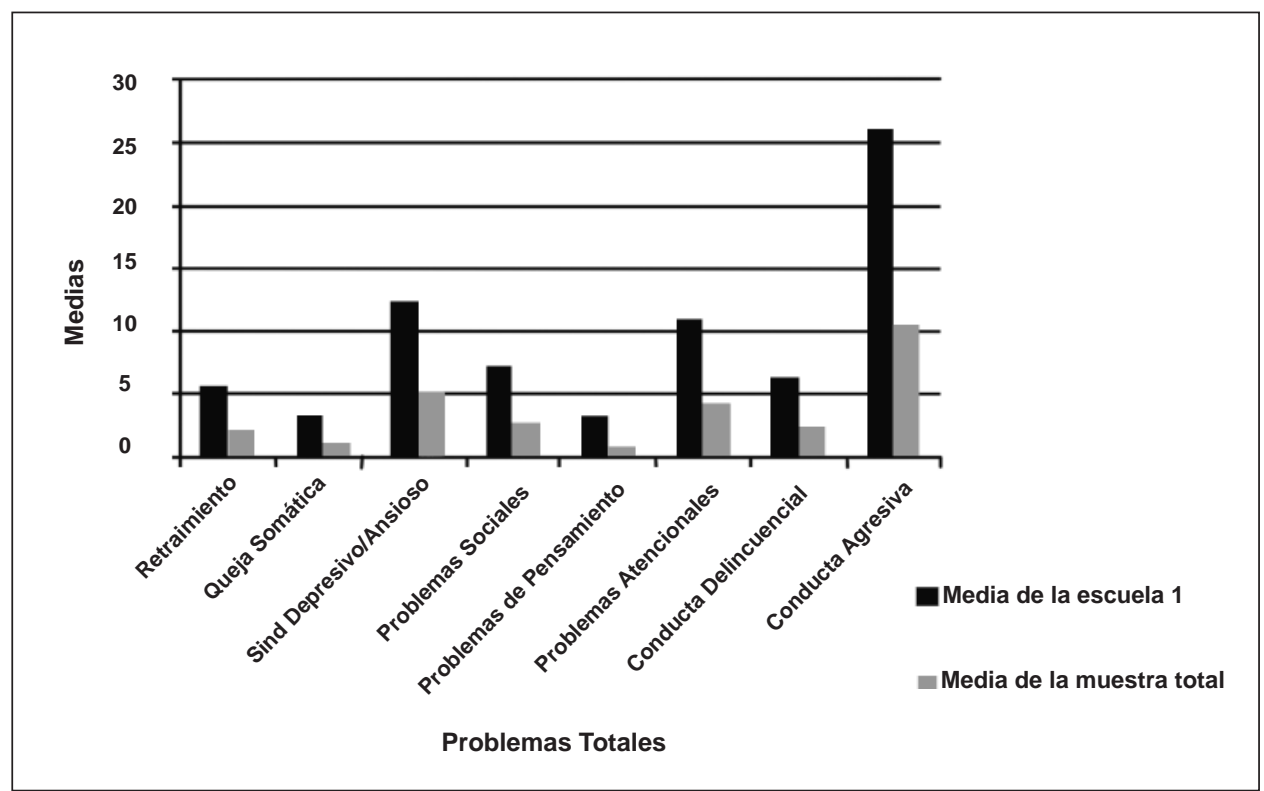

Figura 2. Perfil de niños con rango clínico en problemas emocionales y conductuales, Escuela 1 (Nivel socioeconómico bajo)

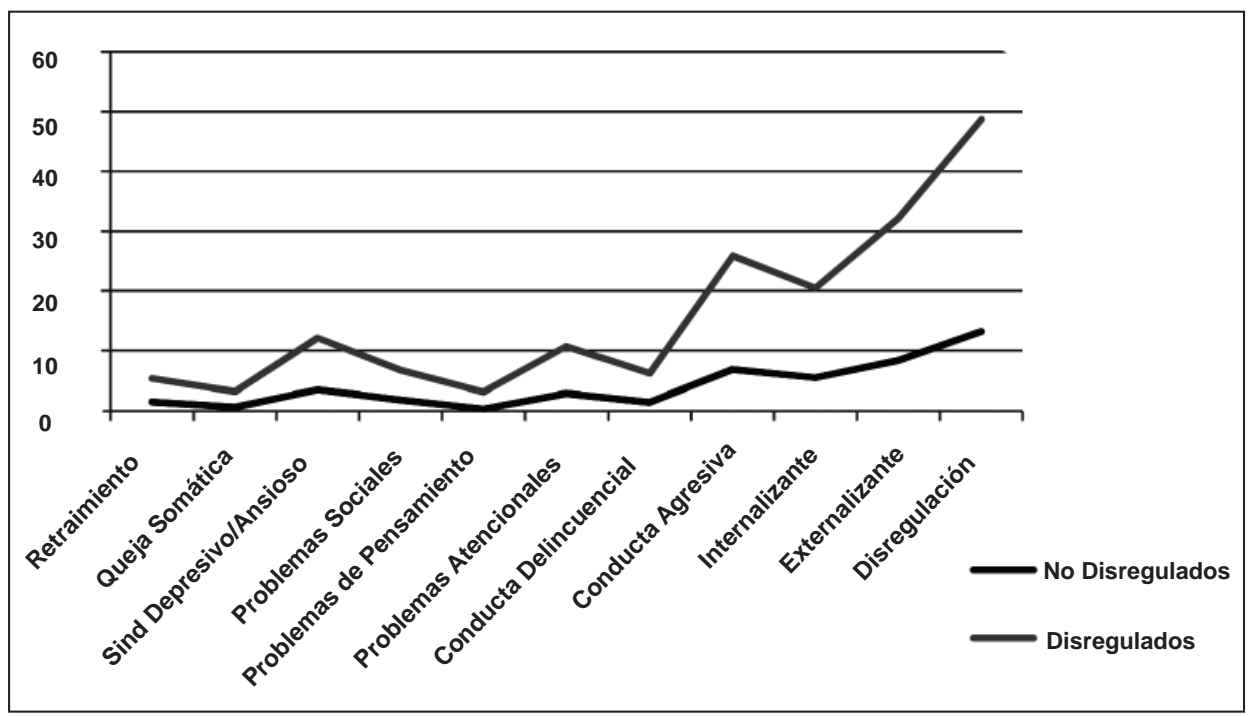

Figura 3. Cuadro comparativo de puntuaciones medias obtenidas por niños disregulados y no disregulados de acuerdo al CBCL 
Por último quisiera mencionar los resultados obtenidos a partir de la prueba de conductancia dérmica en la que se evidencia que los niños con conducta delincuencial reaccionan menos frente a todos los estímulos y en especial frente a los que representan imágenes temibles (Figura 4).

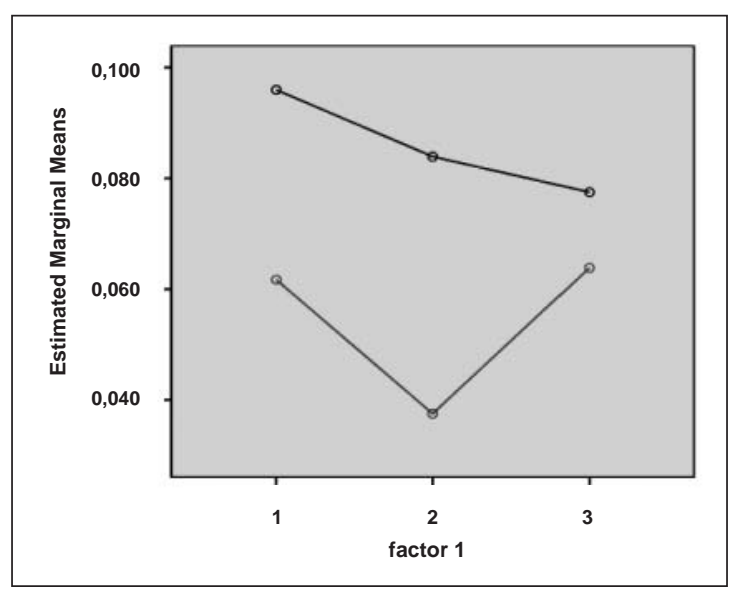

Figura 4. Cuadro comparativo de la activación electrodérmica de los niños con conducta delincuencial

\subsection{Evaluación cualitativa del juego de los niños disregulados}

Comparando el juego de los niños disregulados (identificados por los resultados del Child Behavior Check List) con los no disregulados, en ambas pruebas (FIRE y McArthur Story Stem Battery), podemos concluir que los primeros evidencian un nivel menor de complejidad y creatividad tanto en las acciones de juego como en la narrativa que lo sostiene, lo que genera producciones en general más pobres, con contenidos repetitivos, un juego más apegado a una situación concreta, en general agresiva, en la que no aparecen claras las diferencias entre buenos y malos, amigos y enemigos, acciones efectivas o evitativas.

Las reacciones tanto a las consignas como entre los personajes del juego suelen ser inconsistentes y varían sin motivo. Evidencian un lenguaje escaso y pobre y muy escasa capacidad de exploración.El juego se vuelve, entonces, repetitivo e impermeable a las alternativas planteadas por el entrevistador.

La intensidad de los afectos aumenta en los momentos de tensión (incendio o dilemas) por lo que las situaciones estresantes suelen ser negadas y en consecuencia las alternativas de solución resultan mágicas o son ineficaces. Los patrones cognitivos de funcionamiento se ven alterados por esta invasión de la afectividad y pierden en consecuencia su eficacia como reguladores. Los afectos evidenciados son predominantemente negativos, resultando llamativa la ausencia de miedo y tristeza. La capacidad para reconocer, regular, vivenciar, comunicar y expresar afectos está claramente disminuida, y por lo general el desborde afectivo adquiere tal magnitud que desorganiza también el comportamiento. La agresividad evidenciada suele ser altamente destructiva, no apareciendo como reacción al ataque sino previamente, ya que lo supone. Aparecen con gran frecuencia personajes o situaciones de alto contenido persecutorio frente a las cuales oscilan entre mostrarse omnipotentes o desvalidos, pero generalmente poco efectivos.

Las representaciones de sí resultan poco consistentes, primitivas, esquizoides, cargadas de contradicciones con respecto a sus condiciones y fortaleza. De la misma manera parece haber cierta dificultad en la autoobservación y la observación y comprensión de los otros, evidenciando escasa empatía. La conflictiva expresada hace referencia tanto a una imagen desvalorizada de sí mismo como a impulsos (agresividad) que no logran controlar adecuadamente (conflictiva entre el deseo y la exigencia moral) así como a necesidades básicas insatisfechas, todo lo cual genera dificultades a nivel de la regulación afectiva. Los patrones conflictivos disfuncionales parecen centrarse en torno al eje sumisión-control así como al valor del self versus el valor del objeto. Evidenciando modos pasivos de elaboración (OPD Task Force, 2008). Resultan muy escasas las referencias a valores morales.

No logran manejar adecuadamente la ansiedad que les genera la situación de entrevista o de juego. Suele ser una ansiedad excesiva, persecutoria, que altera el sentido de realidad (no así el juicio) y en consecuencia no resulta efectiva en el control de la situación ansiógena, ya que no corresponde con el estímulo que la dispara. Esto se potencia aún más frente al juego libre.

Los mecanismos de defensa evidenciados son predominantemente primarios (control omnipotente, identificación proyectiva, idealización, negación, proyección), apareciendo como mecanismo secundario fundamentalmente la regresión. 
El funcionamiento mental en general presenta una tendencia a la rigidez y genera patrones relacionales claramente disfuncionales: perciben a los otros como potenciales atacantes, se defienden de este supuesto ataque atacando ellos y en consecuencia generan molestia, enojo e incomprensión en los demás. Esto confirma su impresión de no ser valorados, de ser agredidos y entonces agreden. No evidencian empatía sino que las situaciones que plantean oscilan entre la retracción y el distanciamiento, evitando toda intimidad.

Contratransferencialmente suelen generar en el entrevistador reacciones en las que predomina la molestia, la extrañeza o el aburrimiento.

A través de la caracterización de los personajes del juego manifiestan una escasa conciencia de esta dinámica, evidenciando solamente enojo e incomodidad, sin poder tomar contacto con la vivencia de desvalimiento e inseguridad que puede estar en la base, lo que los lleva a mantenerse a la defensiva así como a atribuir las responsabilidades a otros y repetir y confirmar la situación una y otra vez.

A nivel de diagnóstico podemos entonces inferir en estos niños una perturbación de moderada a severa en el nivel de funcionamiento de la personalidad (American Psychiatric Association, 2011), restricciones y alteraciones mayores en el funcionamiento mental (PDM Task Force, 2006) y un nivel de integración de moderado a bajo en lo que respecta a la estructura (Grupo de Trabajo OPD, 2008), pudiendo estas condiciones darse en diferentes cuadros psicopatológicos, orientándose hacia la generación de un nivel de funcionamiento de personalidad border (PDM Task Force, 2006).

Estas vulnerabilidades, que tienden a hacerse permanentes, generan una especial fragilidad en la cual resulta difícil diagnosticar una conflictiva específica ya que parece predominar la falla en las funciones, constituyéndose una patología estructural más de carencia que de conflicto.

\section{Discusión}

La muestra, seleccionada por conveniencia, quedó conformada por 82 niños de Montevideo, representantes de medios socioeconómicos diferentes (bajo, medio y alto), La media de edad fue 6,2 años, con una prevalencia del $64 \%$ de varones.
Con respecto a la constitución familiar encontré que la Escuela 1 (población de más bajos recursos socio-económico-culturales) evidencia la más alta presencia de hogares monoparentales (44\%), frente a la escuela $2(27 \%)$ y la 3 (14\%). También presenta la más alta frecuencia de antecedentes psiquiátricos (33\%) con una llamativa diferencia frente a las otras dos escuelas ( $8 \%$ y $5 \%$ ), resultando en este caso una diferencia significativa estadísticamente. Ello nos habla de condiciones muy desfavorables para el desarrollo infantil en los hogares de más bajos recursos: la sobrecarga de la persona que se hace cargo de la familia (generalmente madre adolescente con varios hijos) y la fuerte presencia de alteraciones mentales (con la carga genética y la incidencia en el funcionamiento diario que implican). Todo ello podrá favorecer la aparición de mayor disregulación (como lo evidencié en los resultados) tanto desde el lado de la constitución de estos niños como desde la perspectiva de cuidadores poco disponibles en función de su propia condición así como de la sobrecarga.

Aquí se encuentran dos aspectos de las series complementarias (aspectos constitucionales y experiencias infantiles) que estarían incidiendo fuertemente en la consolidación de una disposición caracterológica crónica hacia la disregulación. Y más aún si se piensa que esta disposición en interacción con ese entorno, seguramente favorecerá factores accidentales de valor negativo y se podrá entonces desarrollar un trastorno afectivo, o aún más un trastorno de personalidad.

Al relacionar ahora las características mencionadas (edad, sexo, composición familiar) con el nivel de desarrollo esperado para la muestra: si se considera solamente la media de edad se podría esperar una capacidad reflexiva ya instaurada, con una buena posibilidad de regulación afectiva y basada en el uso de herramientas cognitivas secundarias. En especial se esperaría que las reacciones de estos niños correspondieran en buena medida a estrategias internalizadas, de las cuales los niños tuvieran cierta conciencia y una posibilidad de dominio. Ello no descartaría que los modelos del entorno tuvieran un papel muy importante, pero igualmente tanto la regulación de los afectos como las herramientas cognitivas se verían más afectadas en los niños pertenecientes a medios socioeconómicos más deprivados, lo cual también echaría luz sobre las conclusiones del estudio retrospectivo que 
motivó esta investigación. De acuerdo a esto se esperaría un funcionamiento general más sano en la escuela 3 (perteneciente a un medio socioeconómico alto), algo menos en la Escuela 2 (nivel socioeconómico medio) y notoriamente menor la Escuela 1 (nivel socioeconómico bajo).

Por un lado vemos que es justamente en la Escuela 1 en donde encontré mayor número de problemas afectivos y de enseñanza, así como de síndromes internalizantes, externalizantes y de disregulación. Todo esto también tiene su corroboración al comprobar que justamente dicha escuela presenta diferencias significativas con las otras dos escuelas en todas las escalas del CBCL y del TRF, resultando en todos los casos la que evidencia un nivel mayor de problemas reportados.

Claramente dicha escuela tiene los más altos índices de niños en rango clínico en todos los ítems evaluados, especialmente en Retraimiento, Problemas sociales, Problemas atencionales, Trastornos Internalizantes, Externalizantes y Escala de Disregulación. Algo menos, pero igualmente significativo en el síndrome Ansioso Depresivo.

Al profundizar en cuáles serían esos problemas informados por los padres (Figura 1) vemos que destaca claramente la agresividad, el síndrome depresivo-ansioso y los problemas atencionales, que son justamente los indicadores de disregulación de acuerdo a los estudios de Althoff, Ayer y Hudziak (2010).

En la escuela 1, al analizar el perfil de los niños con rango clínico (Figura 2) nos encontramos con que predomina la conducta agresiva y el síndrome depresivo ansioso, lo cual por un lado evidenciaría aspectos que integran la escala de disregulación y por otro nos mostrarían los dos polos en los cuales la disregulación puede expresarse, o bien a través de una fuerte necesidad de defensa y ataque (síndrome de externalización) o bien a través del desgano, la tristeza o la inhibición (síndrome de internalización), todos los cuales pueden ser indicadores de desvalimiento y vulnerabilidad difiriendo solamente en la forma de tramitarlos. Esta figura nos evidencia nuevamente la alta correlación entre el medio socio económico y los problemas de comportamiento.

Con respecto a la disregulación nos encontramos en primer lugar con una prevalencia del 18,9\% de niños con Disregulación (+ de 1 $D E)$, de los cuales el $64 \%$ son varones mientras solamente el $36 \%$ son niñas. Esto sería coinci- dente con lo esperable, una alta presencia de disregulación y en especial entre los varones. Los niños disregulados aparecen con más problemas emocionales y conductuales que los no disregulados en TODAS las escalas del CBCL (Figura 3). Esto sería una evidencia de la alta incidencia de la regulación emocional en el desarrollo y en la organización de la personalidad.

Pruebas proyectivas (FIRE y MSSB): Estos instrumentos fueron incluidos en la investigación con el objetivo de profundizar en las vivencias subjetivas del niño para relacionarlas con su propia disregulación. Me interesa especialmente adentrarnos en el mundo interno del niño en busca de la dinámica consciente o inconsciente que da lugar a sus manifestaciones. Las técnicas seleccionadas toman en consideración dos situaciones diferentes. El FIRE es una propuesta de juego libre y el MSSB supone un juego estructurado en base a una consigna que incluye un dilema específico que será el disparador para la respuesta del niño. Ambas incluyen la consideración de la narrativa que acompaña el juego.

Estas características de las técnicas marcaron ya una primera diferencia entre los niños disregulados y los no disregulados. Los primeros evidenciaron una desorganización mayor cuánto más libre era la consigna y más aún frente a la combinación de libertad en la expresión a través del juego y la presencia de un estresor. Es decir, los niños disregulados obtuvieron perfomances más pobres en el FIRE y más aún frente a la presencia de la consigna de incendio. Es como si librados a sus propias motivaciones y fantasías los niños disregulados (al no poder identificar un atacante externo del cual defenderse) cayeran en una confusión desorganizativa que les empobrece y rigidiza sus reacciones evidenciando que al no poder defenderse no pueden tampoco reconocerse ni reconocer ciertos afectos que los confundirían aún más (el temor o la tristeza). Esta desorganización aumenta y dispara afectos negativos que no pueden ser equilibrados con afectos positivos.

Esa situación es algo diferente frente a la propuesta de juego más estructurada (MSSB). Frente a un dilema específico pueden poner en juego estrategias de defensa, que aunque también rígidas y no adecuadas, les hacen sentir en el mismo accionar, más organizados; pero curiosamente la consigna más estructurada que permite una respuesta más organizada, 
dispara más afectos positivos pero también más afectos negativos (tristeza o agresividad) que seguramente serán defensivos frente a la conmoción que genera un mayor contacto con sus propios impulsos, deseos, pensamientos y las posibilidades de modularlos. Entonces en definitiva la diferencia no resulta en un nivel de desempeño más adecuado y las representaciones de sí resultan escasamente integradas o consistentes.

La imposibilidad de poder catalogar un estímulo como peligroso o inocuo, y el verse enfrentados a la vez a sus propios recursos y fantasías, como sería el caso de una consigna libre de juego, tiende a desorganizar a los niños con disregulación y esa podría ser la razón para el deficiente manejo de los afectos.

Así nos encontramos con que en ambas técnicas coinciden las evidencias respecto al uso empobrecido de las herramientas para la regulación de los afectos, y en especial del temor y la tristeza. Los niños disregulados evidencian tanto en el FIRE como el MSSB menos temor que los niños no disregulados y con respecto a la tristeza o bien no aparece (FIRE) o es menor en los disregulados que en los regulados (MSSB).

¿Cuál podría ser la razón? En busca de una respuesta recurrí a los planteos de Fonagy et al. (2004), quien dice que los niños con alta disregulación, a quienes el afecto invade y desorganiza, se ven obligados a estar en un estado de alerta que les lleva a desarrollar especiales habilidades de percepción (tanto del entorno como de ellos mismos) así como de detección de situaciones conflictivas. Pero, más allá de este estado de alerta que les permite ser muy agudos en la captación de ciertas señales luego, al no lograr significarlas de forma adecuada, el proceso de mentalización (base de la regulación) se ve afectado, lo que genera la falla en la regulación. Es como si las herramientas cognitivas estuvieran presentes pero al verse invadidas por una afectividad que no logra ser mentalizada dejan de ser operativas.

Algo del mismo tenor podría explicar el bajo nivel de temor: para sentir temor es necesario significar un estímulo como temible, para lo cual es necesario en alguna medida reconocernos a nosotros mismos, reconocer a los demás y en última instancia hacernos cargo de la vulnerabilidad que hace que el estímulo nos resulte temible. Los niños con mayor disregulación al no lograr una adecuada mentalización no llegan a establecer una representación de sí o de los objetos, coherente, adaptada y estable, por lo cual, al no conocerse difícilmente logren aceptar su vulnerabilidad, temer y a la vez organizar alguna estrategia. En vez de este procesamiento actúan impulsivamente o agreden. También se puede inferir que si pudieran registrar el temor eso los haría sentir mucho más vulnerables y es entonces una defensa el inhibir el proceso de mentalización que podría llevar a ello.

Lo mismo sucedería en el caso de la tristeza.

Desde el punto de vista psicofisiológico (Figura 4) se comprobó que uno de los rasgos característicos de los sujetos con alta agresividad es la hiporesponsividad del sistema nervioso autónomo ante estímulos de carácter emocional (Raine, Venables \& Williams, 1995). ¿Corresponderá esto a un déficit en el procesamiento emocional, a una inhibición de la respuesta ante estímulos amenazadores y en consecuencia a una inhibición del temor? ¿Será que en realidad no le dan a esos estímulos el valor que se supone que tienen, no interpretando como temible o como sufrimiento ajeno lo que parecería indicarlo?

Se puede pensar que efectivamente en ellos se da un funcionamiento anómalo de las estructuras que valoran emocionalmente al estímulo, especialmente la amígdala y la corteza prefrontal, lo cual estaría impidiendo la asociación entre un estímulo potencialmente amenazador y la reacción fisiológica.

Si unimos esto con lo propuesto por Blair (1999) acerca de la inhibición del mecanismo de violencia que sugiere que el déficit primario es un mal funcionamiento del sistema neurocognitivo involucrado en la respuesta a las señales de sufrimiento ajeno, que en personas normales funciona como inhibidor conductual, nos podría dar luz acerca de la escasa empatía que evidenciaron estos niños de acuerdo a nuestro análisis de las técnicas proyectivas.

Estas reflexiones me orientan hacia la importancia de identificar ciertos marcadores, aún cuando estos puedan provenir de diferentes perspectivas, que nos ayuden especialmente a la prevención de dinámicas psíquicas disreguladas, al comprobar las consecuencias que la disregulación afectiva tiene sobre el desarrollo.

Vayamos ahora a la consideración de la evaluación cualitativa, lo primero que quisiera destacar es el sentimiento contratransferencial que causó la evidencia de disregulación tanto 
en la aplicación de los instrumentos como en su evaluación. Estos niños que tenían producciones pobres, repetitivas, muy lineales, con una fuerte agresividad que era difícil de comprender en su magnitud, la dificultad para dar soluciones efectivas tanto a los dilemas como al juego libre (en el que se desorganizaban aún más), generaban una sensación de asombro al comienzo pero de aburrimiento después y de resultar poco útiles o importantes para el niño.

Esto solía generar un deseo menor de participación y lentamente iba menoscabando la empatía lo que generaba un notorio distanciamiento. ¿Qué sucederá entonces con el entorno que rodea a estos niños? Y no me refiero solamente al entorno más próximo, en el cual seguramente reciban escasa atención, sino por ejemplo al entorno escolar. ¿Podrán establecer fuera de casa vínculos diferentes, en los que ellos sean tratados de forma diferente y en consecuencia puedan operar como bases seguras que generen apegos seguros? O es que en la medida en que la disregulación desorganiza, empobrece y distancia de los demás lo esperable será que se repitan las experiencias de desvalorización, de abandono, de soledad, no permitiendo una resignificación de las representaciones de sí o del objeto y generando un relacionamiento objetal pobre, escaso, poco empático.

La afectividad no actúa como señal, en cambio invade y al no ser adecuadamente significada se rigidiza: los afectos son siempre los mismos y de valor negativo. El pensamiento se empobrece y no estimula ni genera intereses. Esto parece estar evidenciado en la falta de exploración ya que los niños no dedicaban un tiempo a reconocer los juguetes para luego armar su juego, se lanzaban a la acción sin la posibilidad de selección. Esto corresponde con lo que pasa en el diario vivir: los niños disregulados son impulsivos, agresivos, prestan poca atención a la vez que evidencian desgano, desvalorización. Como si ellos se sintieran frente a sí mismos igual que nos podemos sentir nosotros al trabajar con ellos.

A su vez cuando se les planteaban situaciones conflictivas o estresantes no las significaban como tales y en consecuencia no llegaban a soluciones adecuadas, lo cual no les permitía hacer una valoración positiva de ellos mismos favoreciendo representaciones de sí muy poco consistentes.

Esta escasa elaboración no permitía que las experiencias se fueran diferenciando y clasifi- cando con las inevitables consecuencias sobre la formación de valores. No parecían tener conciencia de lo que les sucedía y entonces, tal como hacían con los dilemas o las consignas, no lo enfrentaban sino que lo repetían.

En la búsqueda de una vivencia subjetiva encontramos con que a más disregulación menos representación, menos ligazón, más pobreza interior, más vulnerabilidad.

Más que a un sentimiento de desvalimiento parece que fueran susceptibles a los indicadores de vulnerabilidad, a lo que puede resultar potencialmente peligroso, o sea ansiosos, pero incapaces de usar esa ansiedad como una señal que dispare un procesamiento. Quedan como atrapados entre la alta sensibilidad al peligro potencial y la necesidad de una respuesta inmediata que pueda dar la ilusión de eliminar el peligro. Pero luego estas reacciones, al no tener fundamento y en especial al no tener en cuenta no solamente lo que pasa dentro de ellos sino las consecuencias que pueden generar, suelen aumentar la conflictividad en lugar de disminuirla.

En la medida en que estas reacciones se repiten van consolidando un mecanismo que de ser una forma de enfrentar un conflicto pasa a ser una característica estructural, especialmente en el sentido de ir generando un determinado funcionamiento mental que altera las funciones a la vez que consolida las carencias.

Entonces, ¿a qué tipo de trastorno puede ir asociada la disregulación de la afectividad? ¿estaremos hablando principalmente de un Trastorno del humor (por ejemplo Bipolaridad o Depresión enmascarada), o la disregulación generará tal efecto en el desarrollo que podrá consolidarse en un Trastorno de Personalidad?

\section{Conclusiones}

Podemos considerar, luego de este estudio, que el denominador común es que la disregulación de los afectos, expresada de una u otra manera (internalizante o externalizante), desorganiza al sujeto sobre el que genera consecuencias graves que tienden a establecerse y expandirse, pudiendo provocar una inestabilidad característica de las organizaciones limítrofes de personalidad, en las que la percepción de sí mismo y de los otros es cambiante, y se puede manifestar alternativamente de forma internalizante (ansiedad, desvalimiento, depresión) o externalizante (agresividad y oposicionismo), gene- 
rando vínculos caóticos e inconsistentes, muchas veces dañinos para otros o para ellos mismos.

A nivel de diagnóstico y haciendo referencia a los sistemas de diagnóstico actuales, puedo inferir en estos niños una perturbación de moderada a severa en el nivel de funcionamiento de la personalidad (de acuerdo a las conclusiones a las que se va arribando en la elaboración del DSM 5, American Psychiatric Association, 2011), restricciones y alteraciones mayores en el funcionamiento mental (PDM Task Force, 2006) y un nivel de integración de moderado a bajo en lo que respecta a la estructura (Grupo de Trabajo OPD, 2008), pudiendo estas condiciones darse en diferentes cuadros psicopatológicos, orientándose como mencionaba anteriormente, hacia la generación de un nivel de funcionamiento de personalidad border (PDM Task Force, 2006).

Sin embargo no considero la disregulación como un síndrome específico sino como una condición subyacente, dimensional, que condicionará el funcionamiento mental del sujeto pudiendo aparecer en diferentes síntomas o síndromes, como rasgo o como estado, y con diferente intensidad y consecuencias tanto para las manifestaciones comportamentales como para la organización psíquica.

Considerando, a partir del análisis de las correlaciones de los datos descritos anteriormente, cómo se vincula la disregulación de los afectos con diferentes aspectos del desarrollo, podemos confirmar el riesgo que supone la disregulación emocional, considerando que la severidad generalizada es el más importante predictor aislado de la disfunción concurrente y prospectiva (American Psychiatric Association, 2011).

Nos preguntamos, ¿qué es lo que falla en los niños disregulados?; ¿la capacidad afectiva?; ¿las herramientas cognitivas?; ¿la posibilidad de representar, ligar, asociar?; ¿son las experiencias vividas demasiado fuertes, traumáticas, y por eso no pueden ser procesadas, o es el sujeto tan débil que no las puede procesar? Podría ahora decir que la conducta disruptiva resulta la carta de presentación de un self escasamente organizado, generador de una actitud de alerta que favorece una interpretación errónea de los distintos estímulos y vivencias. Esta interpretación genera redes representacionales pobres y rígidas, que son alimentadas por una afectividad distorsionada en la que la natural ansiedad frente a los estímulos que debería de desplegar un proceso de mentalización que contribuyera a una respuesta adaptada (al entorno y a sí mismo) no puede significarse de manera adecuada. Están, por ejemplo excluidos tanto el temor (que implicaría aceptar el propio desvalimiento así como la fuerza del estímulo) como los afectos positivos (que evidenciarían confianza en el enfrentamiento). La depresión se haría entonces muy presente.

Por otro lado este proceso, que iría de la mano con dificultades atencionales correlacionaría con un pensamiento que se va distorsionando. Nos enfrentamos entonces a herramientas afectivas y cognitivas empobrecidas, un estado de alerta natural (en parte relacionado a las herramientas escasas y a su uso poco flexible y en parte a las condiciones que el mismo niño recibe o genera en el entorno) que conduce a un procesamiento mental fallido que no genera confianza ni seguridad, del cual, además, el niño no es consciente lo que dificulta aún más su posibilidad de manejo.

Estos niños, impedidos de un adecuado uso de sus condiciones psíquicas, no logran ni inhibir ni expresar de forma regulada, se vuelcan en acciones cuyo significado y consecuencias parecen desconocer. No buscan ayuda (porque parecen no registrar que la necesitan) ni tampoco generan que la ayuda les sea ofrecida (ya que resultan extraños, no amigables y en alguna medida incomprensibles). Frente a las situaciones conflictivas no logran desarrollar estrategias efectivas a pesar de que parecen comprender cuál es el conflicto. Suelen proponer alternativas que aumentan la conflictividad. Una reacción frecuente es que queden paralizados por el impacto de la situación conflictiva (freezing) y entonces tiendan a negarla o a transformarla mágicamente. Todo esto contribuye a un progresivo deterioro de la imagen de sí, que favorece un desvalimiento que puede ser expresado como tal o compensado con fantasías omnipotentes. Se produce entonces un círculo vicioso del cual les resulta a ellos especialmente difícil salir y a nosotros igualmente difícil establecer el contacto, mantenerlo y sacarlos de ese círculo.

Estas condiciones parecen especialmente favorecidas y promovidas por un entorno de deprivación que incide en su generación y luego en su consolidación, lo cual nos muestra la íntima interrelación entre lo intrapsíquico y lo intersubjetivo en la adquisición de la capacidad de autorregulación. A su vez debemos de tener en cuenta que en el entorno de mayor depriva- 
ción la agresividad suele ser moneda corriente y entonces tanto la disposición constitucional como el entorno refuerzan la manifestación de la agresividad. Si a esto le agregamos que al ser niños que se desconocen, que no conocen suficientemente a los otros, que tienden a defenderse de todo aquello que no comprenden y que a la vez no lo logran procesar de forma adecuada, nos explicamos mejor el porqué de la prevalencia de la agresividad en ese medio, dónde la actuación muchas veces se vuelve imprescindible o inevitable, dónde la hostilidad se vuelve una manifestación dominante de la agresividad ya que se asocia muchas veces a la fortaleza. La destructividad que genera sensación de fortaleza queda unida al placer y otros aspectos sanos de la agresividad como la creatividad, la iniciativa, la superación, quedan entonces claramente desvirtuados. Esa agresividad destructiva, que no conforma al yo ni le atribuye valor, aparece muchas veces asociada a la depresión. Y aquí vemos como se relacionaría la expresión agresiva tanto con la destructividad como con la depresión y la ansiedad.

\section{Referencias}

Achenbach, T.M. (1991). Integrative Guide to the 1991 CBCL/4-18, YSR, and TRF Profiles. Burlington, VT: University of Vermont.

Achenbach, T. M. (1993). Empirically based taxonomy: How to use syndromes and profile types derived from the CBCL/4-18, TRF and YSR. Burlington VT: University of Vermont.

Althoff, R., Ayer, L., \& Hudziak, J. (2010). Assessment of Dysregulated Children Using the Child Behavior Checklist: A Receiver Operating Characteristic Curve Analysis. Psychological Assessment, 22 (3), 609-617. doi: 10.1037/a0019699

Althoff, R., Retew., D., Ayer. L., \& Hudziak, J. (2010). Cross-informant agreement of the dysregulation Profile of the Child Behavior Check List. Psychiatry Research, 178, 550-555.

American Psychiatric Association (2011). DSM-5 Development. Recuperado de http://www.dsm5. org/Pages/Default.aspx

Arnold, M.B. (Ed.). (1970). Feelings and emotions. New York: Academic Press.

Bernardi, R. (setiembre, 2007). Entre el yo y el nosotros. El difícil camino hacia la regulación. Comunicación presentada en las $2^{\circ}$ Jornadas Clínicas. Universidad Católica del Uruguay.

Blair, R. J. (1999). Responsiveness to distress cues in the child with psychopathic tendencies. Personality and Individual Differences, 27(1), 135-145.

Bowlby, J. (1986). Vínculos afectivos. Madrid: Morata.

Bradley, S. (2000). Affect regulation and the development of psychopathology. New York: Guilford Press.
Bretherton, I. (1984). Symbolic play. New York: Academia Press.

Bucci, W. (2003). Varieties of dissociative experiences. A multiple code account and a discussion of Bromberg's case of William. Psychoanalytic Psychology, 20 (3), 542-557.

Cole, P. M., Luby, J., \& Sullivan, M. W. (2008). Emotions and the development of childhood depression: Bridging the gap. Child Development Perspectives, 2, 141-148.

Damasio, A. (2010). El error de Descartes. Barcelona: Plaza.

Emde, R. (1988). Development Terminable and Interminable: Innate and motivational factors from infancy. International Journal of Psychoanalysis, $69,23-42$.

Emde, R., Wolf, D. \& Oppenheim, D. (2003). Revealing the inner worlds of young children. The Macarthur Story Stem Battery and Parent-Child Narratives. New York: Oxford University Press.

Fonagy, P. (2004). Teoría del Apego y Psicoanálisis. Barcelona: Espaxi.

Fonagy, P., Gergely, G., Jurist, E., \& Target, M. (2004). Affect regulation, Mentalization, and the $d>$ evelopment of the self. London: Karnac.

Fonagy, P. \& Target, M. (2002). Psychoanalitic theories. New York: Brunner-Routledge.

Freud, S. (1980). Lo Inconsciente. En J. L. Etcheverry (Trad.), Obras Completas (Vol.XIV, 153-214). Buenos Aires: Amorrortu Editores. (Trabajo original publicado en 1915)

Freud, S. (1980). Inhibición,Síntoma y Angustia. En J. L. Etcheverry (Trad.), Obras Completas (Vol. XX, 71-162). Buenos Aires: Amorrortu Editores. (Trabajo original publicado en 1926).

Green, A. (1999). Acerca de discriminación y la indiscriminación afecto/representación. Psicoanálisis Asociación Psicoanalítica de Buenos Aires, 20 (3), 517-587.

Gross, J. (2007). Handbook of emotion regulation. New York: Guilford Press.

Kernberg, O. (1994). La agresión en las perversiones y en los desórdenes de la personalidad. Buenos Ares: Paidós.

Ledoux, J. (1999) El cerebro emocional. Barcelona: Ariel Planeta.

Miller, D., Aguilar, M., Arrillaga, J., Gioscia, R., Merli, M., y Prego, C. (2009). FIRE, Evaluación de la regulación afectiva a través del juego con animales. Manuscrito inédito. Premio Primitivo Techera a la producción académica (2012). Facultad de Psicología. Universidad Católica del Uruguay.

Grupo de Trabajo OPD (2008). Diagnóstico Psicodinámico Operacionalizado (OPD-2). Barcelona: Herder.

OPD Task Force (Eds.) (2008). Operationalized Psychodynamic Diagnosis OPD-2: Manual of Diagnosis and Treatment Planning. Cambridge, MA: Hogrefe \& Huber.

PDM Task Force (2006). Psychodynamic Diagnostic Manual. Silver Spring, MD: Alliance of Psychoanalytic Organizations.

Piaget, J. (1961). La construcción del símbolo en el niño. México: Fondo de Cultura Económica. 
Raine, A., Venables, P. H., \& Williams, M. (1995). High autonomic arousal and electrodermal orienting at age 15 years as protective factors against criminal behavior al age 29 years. American Journal of Psychiatry, 152, 1595-1600.

Slade, A., \& Wolf, D. P. (1994). Children and developmental approaches to meaning and representation. Nueva York: Oxford University Press.

Sroufe, A. (2000). Desarrollo emocional. La organización de la vida emocional en los primeros años. México: Oxford University Press.
Western, D., Shedler, J., Bradley, B., \& DeFife, J. (2011). An Empirical derived taxonomy for personality Diagnosis:Bridging Science and practice in conceptualizing personality. American Journal of Psychiatry. AiA:1-12

Winnicott, D. (1990). Deprivación y delincuencia. Buenos Aires: Paidós.

Para citar este artículo:

Miller, D. (2013). La regulación afectiva en la conformación de la personalidad. Estudio en escolares de 5 a 8 años. Ciencias Psicológicas VII (1): 7 - 23. 